\title{
THE VEGETATION OF DOVER AND ERITH ISLANDS, KENT GROUP, BASS STRAIT
}

\author{
by J.B. Kirkpatrick
}

(with three tables, one text-figure and four plates)

Kirkpatrick, J.B., 1995 (30:vi): The vegetation of Dover and Erith Islands, Kent Group, Bass Strait. Pap. Proc. R. Soc. Tasm.

129: 25-33. https://doi.org/10.26749/rstpp.129.25 ISSN 0800-7403. Department ofGeography and Environmental

Studies, University ofTasmania, Box 252C, GPO, Hobart, Tasmania, Australia 7001.

The terrestrial vegetation of Dover and Erith Islands is comprised of low forest dominated by Allocasuarina verticillata or Myrtaceac species (including Eucalyptus nitida, Melaleuca ericifolia and Leptospermum laevigatum), closed-scrub, heath, Poa poiformis tussock grassland and a wide variety of coastal vegetation types. While Dover Island, which is infrequently burned and ungrazed by stock, is largely covered by low forest, closed-scrub and heath which almost totally lack exotics, Erith Island, which is frequently burned and grazed by cattle, is largely covered by $P$. poiformis tussock grassland with a high proportion of introduced species. Variation in the less disturbed vegetation of the two islands largely relates to soil nutrient conditions, as indicated by $\mathrm{pH}$, soil drainage and exposure to salt-laden winds. Both islands have significant nature conservation values.

Key words: islands, vegetation, disturbance, edaphic ecology, Bass Strait.

\section{INTRODUCTION}

During the many and protracted periods colder than today that have characterised the Quaternary, Bass Strait was largely dry land. The numerous islands of today provided relative relief in a broad windswept plain. The islands differ markedly in their floras and plant communities (Guiler $e t$ al. 1958, Kirkpatrick et al. 1974, Scarlett et al. 1974), with the presence of particular species and communities providing clues to the nature of the complex migration paths that seem to have occurred as sea level rose. For example, the presence of Eucalyptus globulusssp. pseudoglobulus on Rodondo Island and northern Flinders Island does not fit neatly with environmental gradients (Jordan et al. 1993). The islands also differ markedly in their degree of disturbance by human activities. All were uninhabited when Europeans arrived to settle in Australia. Most islands were subsequently burned and stocked. The few islands that escaped, or largely escaped European disturbance have few exotic plant species, while a substantial proportion of the the flora of the most disturbed islands consists of introduced plants (Kirkpatrick 1993).

Although the vegetation of Dover and Erith Islands (fig. 1) has been described at a general level (Mullet \& Murray-Smith 1967, Marginson \& Murray-Smith 1969), and been collected by John Whinray and others, like most Bass Strait islands, these have escaped thorough vegetation survey. Despite the fact that the two islands are linked at low tide, they contrast markedly in their degree of disturbance. Dover, although occasionally burned, has not been grazed by stock or cleared. Erith has been grazed and frequently burned, in order to replace scrub with grass, since the early days of settlement in Bass Strait. This very different disturbance history can be readily explained by the relatively greater extent of soils formed on calcarenite on Erith and the relative steepness and rockiness of Dover.

This paper reports the results of a quadrat-based vegetation survey of the two islands, explores the environmental relationships of the plant communities and discusses the significance of the vegetation of the two islands for conservation.

\section{METHODS}

The vegetation of the islands was surveyed in March 1994, using $10 \times 10$ m quadrats located to encompass variation in dominance and structure, as perceived on aerial photographs, on several traverses of both islands. A vegetation map was drawn, using these aerial photographs and information from ground survey, including oblique photographs taken in the field.

At each quadrat location the following data were recorded: all observable higher plant species, the height and cover of the dominant stratum, the dominant species, the altitude, slope, aspect, surface $\mathrm{pH}$ and \% rockiness. Aspect was divided into five classes related to the degree of drying to be expected from the sun (1, northwest; 2 , north and west; 3 , northeast and southwest; 4 , south and east; 5 , southeast). Because of the time of year in which sampling took place, some annuals and geophytes, such as Bulbine bulbosa and B. semibarbata and the 15 orchid species known from the islands (Beth Gott, pers. comm.), would have been missed.

The floristic data were sorted using the polythetic divisive programme TWINSPAN (Hill 1979) and nine communities were selected from this matrix. Percentage frequencies of species in each of these groups were calculated. Means, standard deviations, 25 percentiles and ranges were calculated for the quadrats in all groups with more than three individuals, and for species occurring in ten or more quadrats for altitude, slope, aspect, $\mathrm{pH}$, vegetation height and rockiness. Data on dominance was tabulated by floristic community.

Species nomenclature follows Hnatiuk (1990) and structural nomenclature follows Specht (1981).

\section{RESULTS AND DISCUSSION}

\section{Vegetation mapping}

Ten mapping units were discriminated (fig. 1). One unit, Myoporum insulare shrubland/open-scrub, is confined to Erith, where it typically consists of clumps of $2-4 \mathrm{~m}$ tall shrubs of $M$. insulare, separated by areas dominated by 


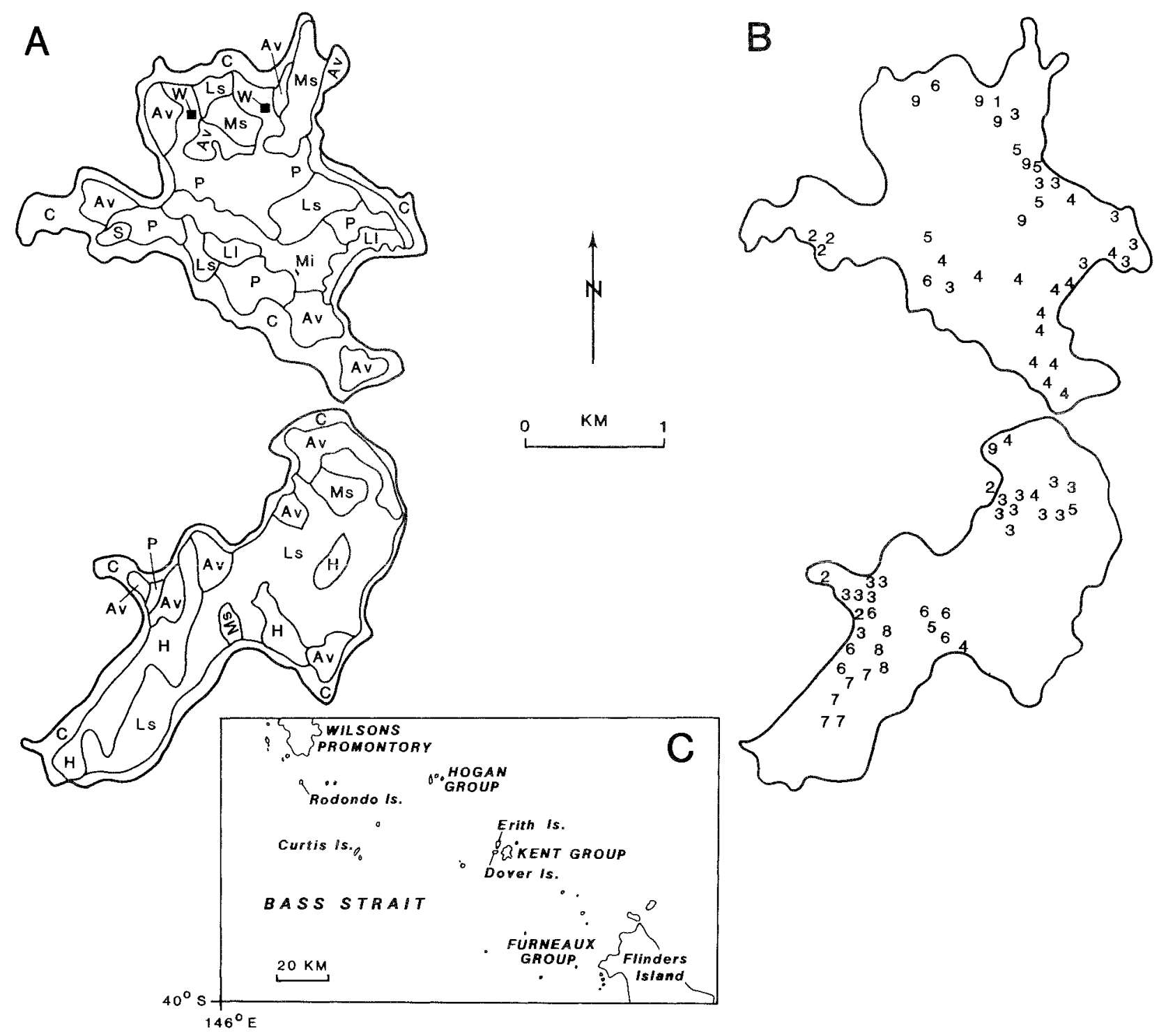

FIG. $I-($ A) Vegetation map. Av = Allocasuarina verticillata open/closed-forest; $C=$ coastal complex; $H=$ heath; $L l=$ Leptospermum laevigatum closed-scrub; $L s=$ Leptospermum scoparium-Kunzea ambigua $c l o s e d-s c r u b ; M i=M y o p o r u m$ insulare shrublandlopen-scrub; $M s=$ Melaleuca ericifolia closed heath/scrublforest; $P=$ Poa poiformis tussock grassland; $S=$ Stipa stipoides tussock grassland, $W=$ wetland. (B) The distribution of quadrats in each classificatory group. (C) The location of the study area and places mentioned in the text.

either Poapoiformis or Lepidospermagladiatum(pl. 1). These latter two species have understories dominated by exotic grasses and herbs. This mapping unit probably represents a stage in the re-invasion of culturally induced $P$. poiformis tussock grassland. $M$. insulare does not burn readily and is not grazed by the cattle present on Erith. The unit is confined to calcareous sands over calcarenite.

Two small wetlands fed by springs in the calcarenite occur on Erith Island. Their vegetation is dominated by Juncus species. The understorey contains a variety of native and exotic herbs (table 1, community 1).

Poa poiformis tussock grassland (pl. 2) is more widespread on Erith than on Dover. It varies in its structure from closed to open, depending on grazing pressure. On Erith Island, the understorey to the tussocks is largely dominated by exotic species. On Dover, the understorey taxa are largely native. Some of the area mapped in this unit has vegetatively resprouting individuals of Melaleuca ericifolia, indicating that, in the absence of further fire, it would become closed-scrub/low closed-forest. On Dover, this vegetation type is only found in situations close to sea level and exposed to considerable salt spray.

Stipa stipoides tussock grassland is widespread around the coasts of both islands but is mostly too localised to be mappable at the scale used. It usually occurs between a zone dominated by succulents and $P$. poiformis tussock grassland. S. stipoides often has a succulent understorey.

The coastal complex includes both rocky shore and foredune vegetation, the latter being confined to Erith, as Dover has no beaches. Dominance is highly variable. On the foredune at Erith, Spinifex sericeus, Austrofectuca littoralis and Helichrysum paralium are variously dominant. Where birds nest, Tetragonia implexicoma is often dominant. On calcareous clifftops, Beyeria leschenaultii, Calocephalus 


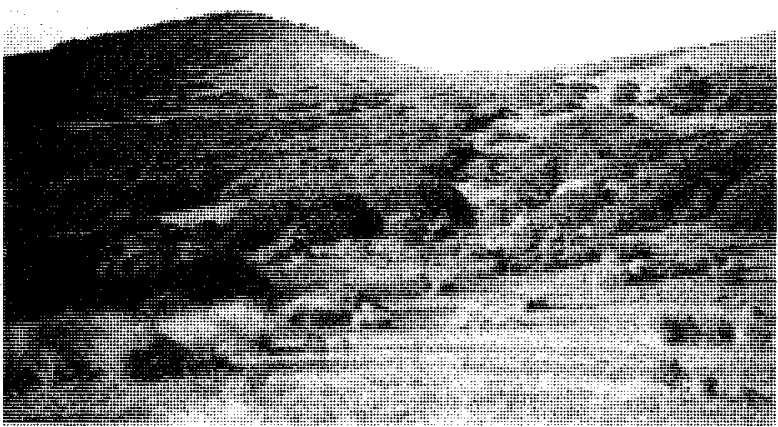

PLATE 1

Myoporum insulare shrubland/open-scrub on Erith Island.

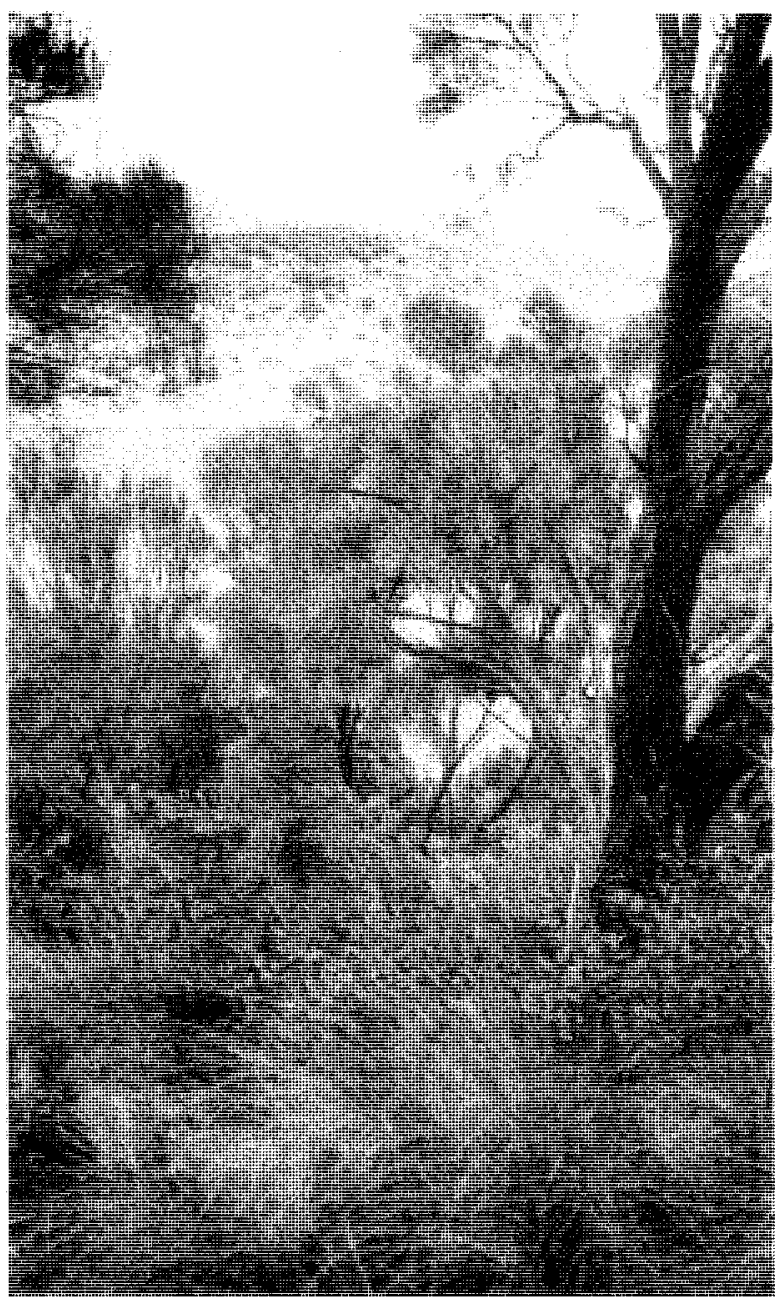

PLATE 3

Allocasuarina verticillata low forest on Erith Island. Deal Island is in the background.

brownii and Pomaderris paniculosa are among the many dominants. In highly exposed granite cracks, Apium insulare or Ixiolaena supina are often dominant. One small area of Sarcocornia quinqueflora succulent herbfield was noted on Erith.

Allocasuarina verticillata dominates open to closed low forest (pls 2,3) on both islands in rocky places sheltered

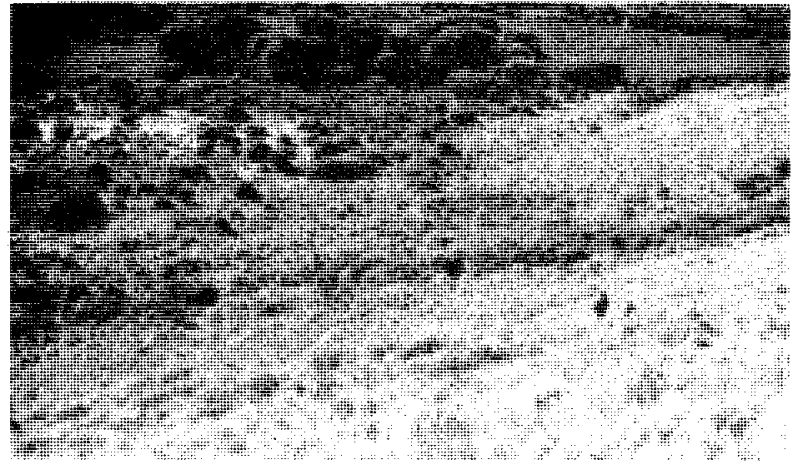

PLATE 2

Poa poiformis tussock grassland on Erith Island on calcarenive with Allocasuarina verticillata low forest and MelaleucaLeptospermum closed-scrub on the granite in the background.

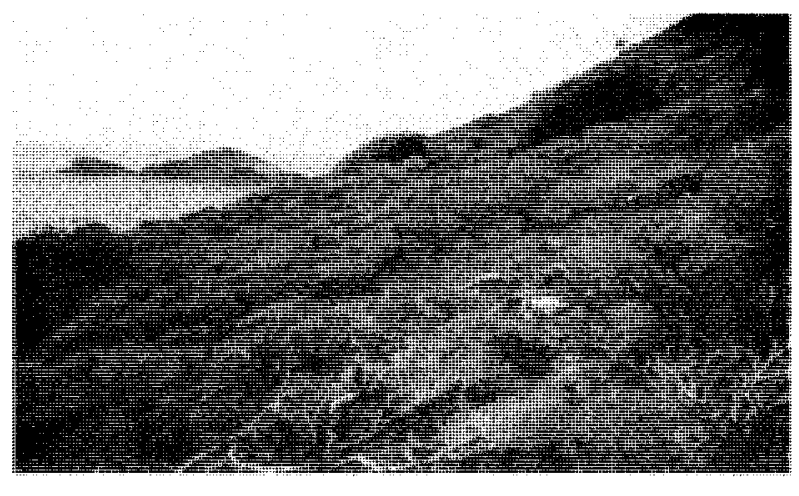

PLATE 4

Heath on Dover Island. The large bushes are Banksia marginata.

from the fiercest of the salt-laden winds and with soils of moderate to high $\mathrm{pH}$. The understorey is highly variable but usually shrubby, although the oldest trees found on Dover had an understorey of dead branches and cladodes entwined with Clematis microphylla.

Three types of closed-scrub/low closed-forest are mapped. The first has substantial cover of Melaleuca ericifolia, which occurs variably with Leptospermum Laevigatum, L. scoparium, Kunzea ambigua, Eucalyptus nitida (not on Erith) and Monotoca elliptica. The second type consists of a closed-scrub/ closed-forest dominated by L. laevigatum, which constitutes almost all of its biomass. This community is confined to calcareous soils on Erith. The third type of closed-scrub/ closed-forest is dominated by varying combinations of L. scoparium, E. nitida (not on Erith), Kunzea ambigua, Callitris rhomboidea and $M$. elliptica. It is found on weakly acid ground on both islands.

The final mapping unit is heath (pl. 4), which varies from open to closed in structure and has highly variable dominance. Some of the more prominent species in the tallest stratum are Callitris rhomboidea, Leptospermum scoparium, Calytrix tetragona, Epacris impressa and Aotus ericoides. It is characteristic of shallow, rocky soils at relatively high altitude. 
TABLE 1

The percentage frequency of species by floristic community and island

\begin{tabular}{|c|c|c|c|c|c|c|c|c|c|c|c|}
\hline \multirow[t]{2}{*}{$\overline{\text { Species }}$} & \multicolumn{9}{|c|}{ Community } & \multicolumn{2}{|c|}{ Island } \\
\hline & 8 & 7 & 5 & 6 & 3 & 9 & 2 & 1 & 4 & Dover & Erith \\
\hline Acacia mucronata & 33 & 20 & 17 & - & - & - & - & - & - & 8 & - \\
\hline Acacia verticillata & - & - & 17 & 12 & - & 17 & - & - & - & - & 8 \\
\hline *Agropyron littoralis & - & - & - & - & - & - & - & - & 6 & - & 3 \\
\hline Agrostis spp. $\dagger$ & - & - & - & - & 9 & - & 33 & - & - & 17 & - \\
\hline *Aira caryophyllea & - & - & 67 & - & 9 & 83 & - & - & 6 & 3 & 31 \\
\hline Allocasuarina monilifera & 100 & 20 & - & - & - & - & - & - & - & 11 & - \\
\hline Allocasuarina verticillata & - & 40 & 67 & 50 & 39 & 33 & - & - & 19 & 34 & 31 \\
\hline Alyxia buxifolia & - & - & 33 & 87 & 74 & 17 & 33 & - & 62 & 66 & 39 \\
\hline * Anagallis arvensis & - & - & - & - & - & 33 & - & - & 6 & - & 8 \\
\hline Aotus ericoides & 100 & 100 & - & - & - & - & - & - & - & 21 & - \\
\hline Apalochlamys spectabilis & - & - & - & - & - & 33 & - & - & - & - & 6 \\
\hline Apium insulare & - & - & - & - & - & - & 50 & - & - & 3 & 6 \\
\hline Apium prostratum & - & - & - & - & - & - & 33 & 100 & 12 & - & 14 \\
\hline *Aster subulata & - & - & - & - & - & - & - & 100 & - & - & 3 \\
\hline *Atriplex sp. & - & - & - & - & - & - & - & 100 & - & - & 3 \\
\hline Austrofestuca littoralis & - & - & - & - & - & - & - & - & 6 & - & 3 \\
\hline Banksia marginata & 33 & - & - & - & - & - & - & - & - & 3 & - \\
\hline Baumea acuta & 100 & 40 & - & 12 & - & - & - & - & - & 16 & - \\
\hline Beyeria leschenaultii & - & - & - & - & 4 & 16 & - & - & 6 & 5 & 3 \\
\hline Brachyscome diversifolia & - & - & 33 & - & 30 & 17 & 33 & - & 25 & 24 & 19 \\
\hline *Bromus diandrus & - & - & - & - & 35 & 17 & - & - & 31 & 18 & 19 \\
\hline${ }^{*}$ Bromus mollis & - & - & - & - & - & - & - & - & 12 & - & 6 \\
\hline${ }^{*}$ Cakile sp. & - & - & - & - & - & - & - & - & 6 & - & 3 \\
\hline Callitris rhomboidea & 67 & 100 & 33 & 37 & - & - & - & - & - & 24 & 8 \\
\hline Calocephalus brownit & - & - & - & - & 4 & - & 67 & - & 50 & 13 & 22 \\
\hline Calytrix tetragona & 100 & 80 & 33 & 62 & 9 & 17 & 17 & - & 12 & 37 & 17 \\
\hline Carpobrotus rossii & - & - & - & 12 & 30 & - & 33 & - & 25 & 16 & 22 \\
\hline *Centaurium sp. & - & - & 33 & 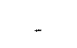 & - & 83 & - & - & 6 & - & 22 \\
\hline${ }^{*}$ Cheiranthus sp. & - & - & - & - & 4 & - & - & - & - & - & 3 \\
\hline Chenopodium glaucum & - & - & - & - & - & - & - & 100 & - & - & 3 \\
\hline Clematis microphylla & - & - & - & - & 18 & - & - & - & 6 & 8 & 6 \\
\hline Comesperma volubile & - & - & - & - & - & 17 & - & - & 12 & 3 & 6 \\
\hline Correa reflexa & - & - & 50 & 50 & 30 & 17 & 33 & - & 75 & 37 & 42 \\
\hline Cotula coronopifolia & - & - & - & - & - & - & - & 100 & - & - & 3 \\
\hline Crassula sieberiana & - & - & 33 & 12 & 4 & - & 50 & - & 6 & 11 & 11 \\
\hline Cyathodes juniperina & - & 40 & 50 & 75 & 35 & - & 17 & - & 12 & 45 & 14 \\
\hline${ }^{*}$ Cynosonus sp. & - & - & 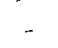 & - & - & - & - & 100 & - & - & 3 \\
\hline *Dactylis glomerata & - & - & - & - & - & - & - & - & 12 & - & 6 \\
\hline Danthonia spp.末 & - & - & 17 & 12 & 4 & 33 & - & - & 12 & 8 & 19 \\
\hline Daucus glochidiatus & & 20 & - & - & - & 17 & - & - & - & 3 & 3 \\
\hline Dianella revoluta & - & - & - & 12 & 35 & 33 & - & - & 19 & 16 & 22 \\
\hline Dichelachne crinita & & - & 17 & - & 4 & 50 & - & - & 12 & - & 19 \\
\hline Dichondra repens & & - & - & - & 13 & 67 & - & - & 56 & - & 44 \\
\hline Dillwynia glaberrima & 33 & - & - & - & - & - & - & - & - & 3 & - \\
\hline Disphyma crassifolium & - & - & - & - & 22 & - & 100 & - & 6 & 21 & 11 \\
\hline Drosera pygmaea & 33 & - & - & 12 & - & - & 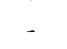 & - & - & 5 & - \\
\hline * Ehrharta erecta & - & - & - & - & 4 & - & - & - & - & - & 3 \\
\hline Epacris impressa & 100 & 80 & - & 37 & - & - & - & - & - & 21 & 6 \\
\hline Epilobium billardierianum & - & - & - & - & - & - & - & 100 & - & - & 3 \\
\hline Eucalyptus nitida & 67 & 60 & - & 12 & - & - & - & - & - & 16 & - \\
\hline Galium australe & - & - & 17 & - & 9 & - & - & - & 6 & 11 & 3 \\
\hline Geranium sp. & - & - & - & - & - & - & - & 19 & - & - & 3 \\
\hline Gnaphalium gymnocephalum & - & - & - & - & - & 33 & - & - & 6 & - & 8 \\
\hline Goodenia ovata & - & - & 83 & 100 & 82 & 67 & - & - & 12 & 58 & 44 \\
\hline Helichrysum dendroideum & - & - & - & - & - & 33 & - & - & - & - & 6 \\
\hline Helichrysum paralium & - & - & - & 25 & 9 & 17 & 33 & - & 37 & 21 & 14 \\
\hline *Holcus lanatus & - & - & - & - & - & 17 & - & - & 6 & - & 6 \\
\hline Isolep is cernua & - & - & - & - & - & - & - & 100 & - & - & 3 \\
\hline Isolepis nodosa & - & - & - & - & - & 67 & 17 & - & 25 & 5 & 19 \\
\hline
\end{tabular}




\begin{tabular}{|c|c|c|c|c|c|c|c|c|c|c|c|}
\hline \multirow[t]{2}{*}{ Species } & \multicolumn{9}{|c|}{ Communicy } & \multicolumn{2}{|c|}{ Island } \\
\hline & 8 & 7 & 5 & 6 & 3 & 9 & 2 & 1 & 4 & Dover & Erith \\
\hline Ixiolaena supina & - & - & - & 12 & - & - & 33 & - & 6 & 3 & 8 \\
\hline Juncus kraussii & - & - & - & 25 & - & - & - & 100 & - & 5 & 3 \\
\hline Juncus pallidus & - & - & - & - & 4 & 17 & - & 100 & - & 3 & 3 \\
\hline Juncus pauciflorus & - & - & - & - & - & 17 & - & 100 & - & - & 3 \\
\hline Kunzea ambigua & - & - & 17 & - & 13 & - & - & - & - & 11 & - \\
\hline Lagenifera stipitata & - & - & - & - & - & 17 & - & - & - & - & 3 \\
\hline *Lagurus ovatus & - & - & - & - & - & - & - & - & 31 & - & 14 \\
\hline${ }^{*}$ Leontodon sp. & - & - & - & - & 4 & 33 & - & - & 31 & - & 22 \\
\hline Lepidosperma concavum & 100 & 100 & - & - & - & - & - & - & - & 21 & - \\
\hline Lepidosperma gladiatum & - & - & - & - & - & 17 & - & - & 50 & 8 & 17 \\
\hline Leptospermum laevigatum & 3 & 100 & 100 & 50 & 30 & 33 & - & - & 25 & 47 & 31 \\
\hline Leptospermum scoparium & 100 & 40 & 67 & 75 & 4 & 50 & - & - & - & 24 & 28 \\
\hline Leucopogon lanceolatus & - & - & 50 & 12 & 13 & 33 & - & - & - & 11 & 14 \\
\hline Leucopogon parviflorus & - & - & 17 & - & 22 & 17 & 17 & - & 69 & 8 & 44 \\
\hline Lilaeopsis polyantha & - & - & - & - & - & - & - & 100 & - & - & 3 \\
\hline Lobelia alata & - & - & - & - & - & - & - & 100 & 19 & - & II \\
\hline${ }^{*}$ Lolium perenne & - & - & - & - & - & 17 & - & - & 19 & - & 11 \\
\hline Melaleuca ericifolia & - & 20 & 83 & 62 & 13 & 33 & - & - & - & 26 & 17 \\
\hline${ }^{*}$ Melilotus indica & - & - & - & - & - & - & - & - & 50 & - & 22 \\
\hline Monotocaspp. & 67 & 100 & - & 37 & - & - & - & - & - & 27 & - \\
\hline Myoporum insulare & - & - & - & - & 30 & - & - & - & 19 & 11 & 17 \\
\hline Olearia phlogoppapa & - & - & 17 & 12 & 78 & 33 & - & - & 31 & 47 & 25 \\
\hline Olearia ramulosa & - & - & 17 & - & - & 50 & - & - & - & - & 11 \\
\hline Olearia viscosa & - & - & - & - & 9 & - & - & - & - & 3 & 3 \\
\hline Oxalis perennans & - & - & - & - & - & - & - & - & 6 & - & 3 \\
\hline Parietaria debilis & - & - & - & - & - & 17 & - & - & - & - & 3 \\
\hline Pimelea linifolia & 67 & 40 & - & 37 & 4 & - & - & - & 6 & 13 & 11 \\
\hline *Plantago coronopus & - & - & 17 & - & - & 50 & - & 100 & 6 & - & 17 \\
\hline Poa poiformis & - & - & 83 & 62 & 70 & 100 & 33 & - & 56 & 50 & 67 \\
\hline Pomaderris paniculosa & - & - & - & - & - & 17 & - & - & 12 & 5 & 3 \\
\hline Pseudognaphalium luteoalbum & - & - & - & - & - & 17 & - & - & 6 & - & 6 \\
\hline Pteridium esculentum & - & - & - & - & 4 & 33 & - & - & 6 & - & 11 \\
\hline Pultenaea daphnoides & 100 & 100 & 67 & 87 & 17 & 17 & - & - & - & 42 & 22 \\
\hline Rhagodia candolleana & - & - & - & - & 70 & - & 17 & - & 69 & 34 & 42 \\
\hline Sambucus gaudichaudiana & - & - & - & - & 4 & - & - & - & - & 3 & - \\
\hline Sarcocornia quinqueflora & - & - & - & - & - & - & 33 & - & - & - & 6 \\
\hline Seneciosp. & - & - & - & - & 4 & 50 & - & - & - & 3 & 8 \\
\hline * Senecio elegans & - & - & - & - & - & - & - & - & 19 & - & 8 \\
\hline Senecio lautus & - & - & - & - & 13 & - & 17 & - & - & - & 11 \\
\hline Solanum sp. & - & - & - & - & - & - & - & - & 6 & - & 3 \\
\hline${ }^{*}$ Sonchus asper & - & - & - & - & 22 & 17 & 33 & - & 56 & 13 & 33 \\
\hline Sonchus megalocarpus & - & - & - & - & - & - & - & - & 6 & - & 3 \\
\hline *Sonchus oleraceus & - & - & - & - & 4 & - & - & - & 12 & - & 8 \\
\hline Spinifex sericeus & - & - & - & - & - & - & - & - & 6 & - & 3 \\
\hline Stackhousia monogyna & - & - & - & - & - & 17 & - & - & 6 & - & 6 \\
\hline Stipa flavescens & - & - & - & - & 4 & - & - & - & 25 & - & 14 \\
\hline Stipa stipoides & - & - & - & - & 9 & - & 100 & - & 19 & 16 & 14 \\
\hline Stylidium graminifolium & - & 100 & 17 & 75 & 4 & 17 & 17 & - & - & 32 & 8 \\
\hline Swainsonia lessertifolia & - & - & - & - & - & 17 & - & - & 31 & 3 & 14 \\
\hline Tetragonia implexicoma & - & - & - & - & 48 & - & - & - & 56 & 24 & 31 \\
\hline Threlkeldia diffusa & - & - & - & - & 4 & - & - & - & 25 & 3 & 11 \\
\hline${ }^{*}$ Trifolium dubium & - & - & - & - & - & 67 & - & - & 44 & - & 31 \\
\hline Urtica incisa & - & - & - & - & 4 & - & - & - & 6 & - & 6 \\
\hline${ }^{*}$ Vellereophyton dealbatum & - & - & - & - & 4 & 17 & 67 & - & 6 & 11 & 8 \\
\hline${ }^{*} V u l p i a \mathrm{sp}$. & - & - & 33 & - & 4 & - & - & - & - & 3 & 6 \\
\hline Wahlenbergia sp. & - & - & - & - & - & 17 & - & - & - & - & 3 \\
\hline Zygophyllum billardieri & - & - & - & - & - & - & - & - & 31 & - & 14 \\
\hline
\end{tabular}

* Non-native species.

$\dagger$ Includes Agrostis aemula and A. avenacea. $¥$ Includes Danthonia caespitosa and D. penicillata. 


\section{Characteristics and environments of floristic communities}

The percentage frequency of species in each of the classificatory groups is shown in table 1 . Table 2 gives environmental, species richness and height data. The distributions of dom inant species among communities is shown in table 3 . The distributions of the quadrats in the communities are shown in figure 1 . Species occurring in more than $60 \%$ of the quadrats in a community are listed by lifeform in the descriptions of the communities below.

\section{Juncus rushland}

Introduced grass: Cynosorus echinatus

Native graminoids: Isolepiscernua, Juncus kraussii, J. pallidus, J. pauciflorus

Native herbs: Apium prostratum, Chenopodium glaucum, Cotula coronopifolia, Epilobium billardierianum, Lilaeopsis polyantha, Lobelia alata

Introduced herbs: Aster subulata, Atriplex sp., Plantago coronopus

This community occurred in only one quadrat. However, its environmental differentiation from other communities is strikingly clear. With constant seepage from a permanent spring of water with a $\mathrm{pH}$ of 8.5 , the community occupies a distinct and highly localised environment. It is being damaged by cattle trampling, which has caused extensive puddling. Both areas observed of this community are in a matrix of Poa poiformis tussock grassland.

\section{Stipa stipoides/Calocephalus brownit tussock grassland/shrubland}

Native shrubs: Calocephalus brownit

Native grasses: Stipa stipoides

Native succulents: Disphyma crassifolium

Introduced herbs: Vellereophyton dealbatum

This coastal community is associated with alkaline soils and high inputs of salt spray. Stipa stipoides is the most frequent dominant. It occurs to the seawards of all other communities (fig. 1).

\section{Allocasuarina verticillata-Alyxia buxifolia low open-forest/open-heath}

Native shrubs: Alyxia buxifolia, Goodenia ovata, Olearia phlogopappa

Native succulents: Rhagodia candolleana

Native grasses: Poa poiformis

This coastal community occurs on more acid soils than either of community 2 or 4 , usually on a jumble of granite boulders. Allocasuarina verticillata and Leptospermum laevigatum are the most frequent dominants among a large number.

\section{Leucopogon parviflorus-Rhagodia candolleana open-heath}

Native shrubs: Alyxia buxifolia, Correa reflexa, Leucopogon parviflorus
Native succulent: Rhagodia candolleana

This coastal community usually occurs on more calcareous soils than community 3. Leptospermum laevigatum is the most frequent dominant, although dominance is highly variable.

\section{Allocasuarina verticillata-Leptospermum scoparium} low forest

\section{Native trees: Allocasuarina verticillata}

Native shrubs: Goodenia ovata, Leptospermum laevigatum, L. scoparium, Melaleuca ericifolia, Pultenaea daphnoides Native grasses: Poa poiformis

Introduced grasses: Aira caryophyllea

This community varies from heath to low forest and can be open or closed. It is usually dominated by Leptospermum scoparium or Allocasuarina verticillata. It occurs in the rockiest situations of the non-coastal communities, invariably on slightly acid soils on granite and at lower altitudes than communities 7 and 8 .

\section{Melaleuca ericifolia-Leptospermum scoparium closed-scrub}

Native shrubs: Alyxiabuxifolia, Calytrixtetragona, Cyathodes juniperina, Goodenia ovata, Leptospermum scoparium, Melaleuca ericifolia, Pultenaea daphnoides

Native grasses: Poa poiformis

Native herbs: Stylidium graminifolium

This community forms a dense closed-scrub, mostly dominated by $M$. ericifolia or Allocasuarina verticillata, generally on southerly, steep, rocky slopes on slightly acid soils. It occurs at lower altitude than communities 7 and 8 .

\section{Dry heath}

Native shrubs: Aotus ericoides, Callitris rhomboidea, Calytrix tetragona, Epacris impressa, Leptospermum laevigatum, Monotoca elliptica, Pultenaea daphnoides

Native graminoids: Lepidosperma concavum

Native herbs: Stylidium graminifolium

This open-heath community is usually dominated by Leptospermum scoparium or Eucalyptus nitida. It occurs on the northerly and westerly aspects and ridges of the granite peaks on highly acid and rocky soils. It intergrades with community 8 as drainage becomes more impeded and with community 6 downslope.

\section{Wet heath}

Native shrubs: Allocasuarina monilifera, Aotus ericoides, Callitris rhomboidea, Calytrix tetragona, Epacris impressa, Eucalyptus nitida, Leptospermum scoparium, Monotoca elliptica, Pimelea linifolia, Pultenaea daphnoides

Native graminoids: Baumea acuta, Lepidosperma concavum This open to closed-heath community occurs on acid soils with the organically enriched A horizon, lack of rock cover and gentle slopes that often indicate periods of poor drainage. It intergrades with community 7 as drainage improves, and with community 6 downslope. Different dominant species are found in each of the three quadrats in the group. 
TABLE 2

Means, standard deviations and ranges for environmental and structural variables by community

\begin{tabular}{|c|c|c|c|c|c|c|c|c|c|}
\hline Community & $1(1)$ & $2(6)$ & $3(23)$ & $4(16)$ & $5(6)$ & $6(8)$ & $7(5)$ & $8(3)$ & $9(6)$ \\
\hline $\begin{array}{l}\text { Aspect mean } \\
\text { (s.d.) range }\end{array}$ & 2 & $\begin{array}{c}2.3 \\
(1.4) 1-4\end{array}$ & $\begin{array}{l}2.9 \\
(2.7) 1-5\end{array}$ & $\begin{array}{c}2.94 \\
(0.93) 1-4\end{array}$ & $\begin{array}{l}2.8 \\
(1.6) 1-5\end{array}$ & $\begin{array}{l}3.0 \\
(1.9) 1-5\end{array}$ & $\begin{array}{l}2.4 \\
(1.5) 1-5\end{array}$ & $\begin{array}{c}1.3 \\
(0.5) 1-2\end{array}$ & $\begin{array}{l}2.5 \\
(1.4) 1-5\end{array}$ \\
\hline Slope & 8 & $\begin{array}{c}21.8 \\
(14.6) 3.40\end{array}$ & $\begin{array}{c}15.1 \\
* 8.6) 2-30\end{array}$ & $\begin{array}{c}19.25 \\
(11.22) 3-45\end{array}$ & $\begin{array}{c}15.7 \\
(10.3) 5-34\end{array}$ & $\begin{array}{c}25.7 \\
(8.6) 15.40\end{array}$ & $\begin{array}{c}14.6 \\
(4.6) 10-20\end{array}$ & $\begin{array}{c}9.0 \\
(1.7) 7-10\end{array}$ & $\begin{array}{c}18.3 \\
(8.8) 5-25\end{array}$ \\
\hline $\mathrm{pH}$ & 8 & $\begin{array}{c}7.7 \\
(1.5)^{6-9.5}\end{array}$ & $\begin{array}{c}6.2 \\
(1.1) 4.5-9.5\end{array}$ & $\begin{array}{c}8.7 \\
(1.2) 6-9.5\end{array}$ & $\begin{array}{c}5.0 \\
(0.5) 4.5-5.5\end{array}$ & $\begin{array}{c}5.1 \\
(0.7) 4.5-6.0\end{array}$ & $\begin{array}{c}4.8 \\
(0.4) 4.5-5.5\end{array}$ & $\begin{array}{l}4.5 \\
(0.0)\end{array}$ & $\begin{array}{c}6.7 \\
(1.5) 5.5 .95\end{array}$ \\
\hline Altitude & 40 & $\begin{array}{c}31 \\
\text { (16) } 5-50\end{array}$ & $\begin{array}{c}46 \\
(34) 5-120\end{array}$ & $\begin{array}{c}53 \\
\text { (39) } 2-140\end{array}$ & $\begin{array}{c}87 \\
(31) 60-140\end{array}$ & $\begin{array}{c}92 \\
(28) 50-120\end{array}$ & $\begin{array}{c}172 \\
(45) 120-220\end{array}$ & $\begin{array}{c}150 \\
\text { (10) } 140-160\end{array}$ & $\begin{array}{c}67 \\
\text { (20) } 40-100\end{array}$ \\
\hline Rock cove: & 0 & $\begin{array}{c}40 \\
(41) 0-90\end{array}$ & $\begin{array}{c}24 \\
(24) 0-70\end{array}$ & $\begin{array}{c}1 \% \\
(30) 0-90\end{array}$ & $\begin{array}{c}35 \\
(34) 0-80\end{array}$ & $\begin{array}{c}33 \\
(41) 0-80\end{array}$ & $\begin{array}{c}34 \\
(36) 0-80\end{array}$ & $\begin{array}{c}2 \\
\text { (3) } 0-5\end{array}$ & $\begin{array}{c}12 \\
(12) 0-30\end{array}$ \\
\hline Richness & 12 & $\begin{array}{c}21.8 \\
(14.6) 3-16\end{array}$ & $\begin{array}{c}10.3 \\
(2.4) 6-16\end{array}$ & $\begin{array}{c}15.4 \\
(6.4) 6-32\end{array}$ & $\begin{array}{c}11.8 \\
(2.0) 9-15\end{array}$ & $\begin{array}{c}11.7 \\
(3.0) 6.16\end{array}$ & $\begin{array}{c}12.2 \\
(1.6) 10-14\end{array}$ & $\begin{array}{c}12.3 \\
\text { (1.1) } 11-13\end{array}$ & $\begin{array}{c}17 \\
(4.3) 12-22\end{array}$ \\
\hline $\begin{array}{l}\text { Vegetation } \\
\text { height }\end{array}$ & 0.4 & $\begin{array}{c}0.2 \\
(0.1) 0.1-0.3\end{array}$ & $\begin{array}{c}2.9 \\
(2.7) 0.1-8.0\end{array}$ & $\begin{array}{c}2.0 \\
(2.7) 0.1-8.0\end{array}$ & $\begin{array}{c}2.7 \\
(2.3) 0.4-6.0\end{array}$ & $\begin{array}{c}2.3 \\
(1.4) 0.1-4.0\end{array}$ & $\begin{array}{c}1.0 \\
(1.4) 0.2-3.5\end{array}$ & $\begin{array}{c}1.8 \\
(1.2) 0.5-3.0\end{array}$ & $\begin{array}{c}0.3 \\
(0.1) 0.2-0.5\end{array}$ \\
\hline
\end{tabular}

TABLE 3

Dominants as a percentage of quadrats by classificatory group

\begin{tabular}{|c|c|c|c|c|c|c|c|c|c|}
\hline \multirow[b]{2}{*}{ Dominant species } & \multicolumn{8}{|c|}{ Classificatory group } & \multirow[b]{2}{*}{1} \\
\hline & 8 & 7 & 5 & 6 & 3 & 9 & 2 & 4 & \\
\hline Juncus kraussii & - & - & - & - & - & - &. & - & 100 \\
\hline Helichrysum paralium & - & - & - & - & - & - & - & 6 & - \\
\hline Lepidosperma gladiatum & - & - & - & - & - & - & - & 12 & - \\
\hline Leucopogon parviflorus & - & - & - & - & - & - & - & 6 & - \\
\hline Tetragonia implexicoma & - & - & - & - & - & - & - & 6 & - \\
\hline Beyeria leschenaultii & - & - & - & - & - & - & - & 6 & - \\
\hline Calocephalus brownit & - & - & - & - & 4 & - & - & 6 & - \\
\hline Leptospermum laevigatum & - & - & - & - & 17 & - & - & 19 & - \\
\hline Stipa stipoides & - & - & - & - & 4 & - & 33 & - & - \\
\hline Apium insulare & - & - & - & - & - & - & 17 & - & - \\
\hline Sarcocornia quinqueflora & - & - & - & - & - & - & 17 & - & - \\
\hline No clear dominant & - & - & - & 25 & - & - & 33 & 12 & - \\
\hline Pomaderris paniculosa & - & - & - & - & - & 17 & - & - & - \\
\hline Poa poiformis & - & - & - & - & 13 & 67 & - & - & - \\
\hline Myoporum insulare & - & - & . & - & 9 & - & - & 6 & - \\
\hline Melalenca ericifolia & - & - & - & 25 & 4 & 17 & - & - & - \\
\hline Allocasuarina verticillata & - & - & 33 & 25 & 35 & - & - & 12 & - \\
\hline Alyxia buxifolia & - & - & - & - & 9 & - & - & 6 & - \\
\hline Kunzea ambigua & - & - & 17 & - & 4 & - & - & - & - \\
\hline Leptospermum scoparium & 33 & 40 & 50 & - & - & - & - & - & - \\
\hline Eucalyptus nitida & 33 & 40 & - & 12 & - & - & - & - & - \\
\hline Callitris rhomboidea & - & 20 & - & 12 & - & - & - & - & - \\
\hline Banksia marginata & 33 & - & - & - & - & - & - & - & - \\
\hline
\end{tabular}




\section{Poa poiformis tussock grassland}

Native shrubs: Goodenia ovata

Native grasses: Poa poiformis

Introduced grasses: Aira caryophyllea

Native graminoids: Isolepis nodosa

Native herbs: Dichondra repens

Introduced herbs: Centaurium erytbraea, Trifolium dubium

This community usually consists of an open stratum of $P$. poiformis tussocks, interspersed with $I$. nodosa, and a low understorey dominated by a mixcure of native and introduced herbs. It occurs on slightly acid to alkaline soils with a low cover of outcropping rock. Most of the area of this community has arisen from the activities of graziers and stock.

\section{Ecological relationships}

Both islands consist of an acid, coarse granite which is partly overlain by aeolian calcarenite, probably derived from the Bassian Plain during times of lower sea level than roday. These two parent materials have resulted in the formation of soils of dramatically different $\mathrm{pH}$, with a range from 4.5 to 9.5. Strong southerly to northwesterly winds bearing considerable quantities of salt spray appear to have interacted with parent material and vegetation to create weakly acid, red soils on parts of the lower granite slopes of the island, which contrast markedly with the highly acid, grey-black soils that are found on the higher peaks.

The role of soil conditions in differentiating the vegetation of the island is undoubtedly strong. Communities 1 , 2,4 and 9 are almost totally restricted to the calcarenite or calcareous sands, while communities 5-8 are restricted to the granite. There are many species that are restricted to one of the two substrata. For example, Zygophyllum billardieri and Beyeria leschenaultii are restricted to calcarenite, while Aotus ericoides, Epacris impressa, Eucalyptus nitida and Lepidosperma concavum are restricted to the granite. However, many other species have a wider edaphic range, with Leptospermum laevigatum and Pultenaea daphnoides occurring over the full range of $\mathrm{pH}$.

Within both of the major geological types, there is a differentiation of the vegetation in response to exposure to salt spray. Community 2 occurs closest to the sea on the more exposed coasts, with community 3 occurring inland of it largely on granite, and community 4 occurring inland of it largely on calcarenitc. The strong altitudinal break in community type may partly relate to exposure to salt spray, but is coincident with edaphic conditions, and may also relate to a precipitation gradient.

Apart from the obvious case of community 1, there is some evidence that soil drainage conditions may be important in differentiating the vegetation of the islands. The correlation of different types of heath with slope and soil characteristics provides circumstantial evidence of this phenomenon. Within the scrub and low forest communities, Melaleuca ericifolia tends to be most prominent in the valleys and on the gentler slopes, while Allocasuarina verticillata exhibits the opposite tendencies. However, this pattern may relate as much to tendency to drought or dryness as to the possibilities of impeded drainage.

Disturbance by burning and cattle-grazing appears to have had a strong influence on vegetation patterning, extending the range of tussock grassland at the expense of closed-scrub and low forest communities, and allowing and expediting the invasion of many exotic plants.

\section{Conservation significance of the flora and vegetation}

The flora of the two islands contains only one Tasmanian endemic species, Eucalyptus nitida, and no nationally rare or threatened species. However, it is rich in species that are rare in Tasmania. These species largely fall into two groups: those that are associated with offshore island coastal vegetation in high energy environments throughout southern Australia; those that are associated with highly calcareous soils.

Ixiolatna supina is one of the coastal species. It is also known from Rodondo, Curtis and Deal Islands in Tasmania and is classified as $\mathrm{r} 1$ by Kirkparrick et al. (1991), this notation denoting that it has a distribution of less than 100 $\mathrm{x} 100 \mathrm{~km}$ in Tasmania. It is found in cracks on the granite cliffs near the seaward limit of vegetation on both islands and appears not to be threatened by grazing or other disturbance. Apium insulare, the other coastal rare species is classified as $\mathrm{r} 2$, denoting presence in less than twenty 10 $\mathrm{x} 10 \mathrm{~km}$ grid squares within Tasmania. It occurs in the moister granite cracks on the most salt spray-exposed clifftops of both islands. On Erith Island, it is only found in situations that are inaccessible to cattle.

The rare species associated with calcarenite are all classified as r2. Pomaderris paniculosa and Beyeria leschenaultii are found on calcarenite-covered clifftops on both islands, while the latter is also found on calcareous beach sand on Erith. Both species are regenerating in all the stands visited. Zygophyllum billardieri is a succulent creeper found on both islands, although it is more common on Erith. It was observed in areas that were both heavily used and unused by cattle. Apalochlamys spectabilis was only observed on Erith, where it acts as a disturbance coloniser in grazed open Poa poiformis grassland. Parietaria debilis was most common in the parts of the island with the highest exotic species cover, where it formed part of a closely grazed sward. Leucopogon lanceolatus was the only rare species to occur in the closed-scrub of the islands. It is classified as $\mathrm{r} 2$.

One of the most scientifically interesting aspects of the vegetation of the two islands is the species richness and composition of the heath that is found on the more elevated parts of Dover. With approximately 11 higher plant species per $10 \times 10 \mathrm{~m}$ quadrat, this heath has very low species richness compared to similar heaths on the Tasmanian mainland and the largest Bass Strait islands. The most floristically similar communities recognised for Tasmania have species richnesses of non-geophytic plants that vary around 30 for the same quadrat size (Kirkparrick 1977). With one major exception, Callitris rhomboidea, the species that occur in these heaths are a small subset of the common species in the heaths of the Tasmanian mainland and southern Victoria. Graminoids are noticeably uncommon, with only Lepidosperma concavum and Baumea acuta being noted. Given that this heath community is floristically distinct from all those recognised from Victoria (McMahon et al. 1990) and Tasmania (Kirkpatrick 1977, 1991), and is likely to be confined to the Kent Group, it must be considered an unreserved community. The other communities on the island have analogues reserved elsewhere in Tasmania, although the communities on calcarenite are poorly reserved. 
A striking and unusual feature of the Dover heaths is the widespread presence of a native gymnosperm, Callitris rhomboidea. This species is easily eliminated by two successive fires in a few years (Harris \& Kirkpatrick 1991). Its presence in the heaths and scrubs of the two islands reflects a continued wide spacing of fires since European occupance.

The vegeration and flora of the Kent Group as a whole have considerable biogeographic interest. The islands to the north have distinctly different scrub and forest vegetation, with Melaleuca armillaris replacing $M$. ericifolia on Curtis and Rodondo, and with Eucalyptus globulus ssp. pseudoglobulus being present on Rodondo instead of $E$. nitida, which reaches its northernmost limit in the Kent Group. Most of the islands of equivalent size to the south have been more severely degraded by grazing and fire than any in the Kent Group. None of them have the heath or eucalypt-dominated scrub found on Dover.

One interesting feature of the flora of the islands is the presence, in very low numbers, of two wet sclerophyll forest species, Olearia viscosa and Pomaderris apetala. These are found in coastal scrub on calcareous soils near the beach on Erith. Olearia viscosa was also found in closedscrub on weakly acid soils on Dover. They may be the remnants of a more complex wet sclerophyll forest that could have occurred in the now submerged gorge between Deal and the two islands.

The low species richness of the inland communities compared to their larger island counterparts could relate to the chance extinctions that are predicted by island biogeography theory (MacArthur \& Wilson 1967). This relaxation process has had at least the 6500 years since present sea levels were attained. It could also be the result of elimination of species because of lack of suitable migration paths, as climate changed and sea levels rose between 18000 and 6500 years ago. A third explanation, which would be consistent with the lack of graminoids in the heath, could be a long period in which fire was absent, allowing the heath to become closed-scrub and, thereby, eliminating the habitat for heath species not also adapted to the saltspray environment or cxistence on skeletal granite soils. This latter hypothesis is supported by the observation of Beth Gott (pers. comm.) that lightning strikes are much less common on Dover than on the adjacent Deal Island.

Given the above significant features of the vegetation and flora of the two islands, it would be desirable to make them, along with the remainder of the Kent Group, a Nature Reserve. The virtually untouched Dover Island has some of the highest claims in this regard of any of the unreserved Tasmanian islands. Care would need to be taken on Erith Island to ensure that the colonising rare species were not lost as shrub and tree reinvasion of the Poa grassland proceeded in the absence of stock and anthropogenic fire.

\section{ACKNOWLEDGEMENTS}

I thank Suzie Madigan and Greg Blake for help in the field, Chris Robinson and Christian Bell for getting us there, Alex Buchanan and Dennis Morris for help with plant identifications and Guus van de Geer for drawing the maps and diagrams.

\section{REFERENCES}

Gultr, E.R., SFrvevir, D.L. \& Whils, J.H., 1958: The Fisher Island Field Station - with an account of its principal flora and fauna. Pap. Proc. R. Soc. Tasm. 92: 171-174.

Harris, S. \& KirkPATRICK, J.B., 1991: The distributions, dynamics and ecological differentiation of Callitris species in Tasmania. Aust. J. Bot. 39: 187-202.

HNATUK, R.J., 1990: CENSUS OF AUSTRALIAN VASCULAR PLANTS. AGPS, Canberra.

HII., M.O., 1979: TWINSPAN - A FORTRAN PROGRAM FOR ARRANGING MULTIVARIATE DATA IN AN ORDERED TWO-WAY TABLE BY CLASSIFICATION OF THE INDIVIDUALS AND ATTRIBUTES. Cornell University, New York.

Jordan, G.J., POTTS, B.M., Kirkipatrick, J.B. \& Garimine, C. 1993: Variation in the Eucalyptus globulus complex revisiced. Aust. J. Bot. 41:763-784.

KIRKPATRICK, J.B., 1977: THE DISAPPEARING HEATH - A STUDY OF THE CONSERVATION OF COASTAL HEATH IN NORTH AND EAST TASMANIA AND THE FURNEAUX GROUP. Tasmanian Conservation Trust, Hobart.

KIRKPATRKCK, J.B., 1991: Reservation status of plant communities in Tasmania. In Kirkpatrick, J.B. (Ed.): TASMANIAN NATIVE BUSH: A MANAGEMENT HANDBOOK. Tasmanian Environment Centrc, Hobart: 277-291.

KirKPATRICK, J.B., 1993: Dry coastal ecosystems of southeastern Australia. In Maarel, E. van der (Ed.): ECOSYSTEMS OF THE WORIL. 2B: DRY COASTAL ECOSYSTEMS - AFRICA, AMERICA, ASIA AND OCEANIA. Elsevier, Amsterdam: 273-288.

Kirkpatrick, J.B., Massty, J.S. \& Parsons, R.F., 1974: Natural history of Curtis Island, Bass Strait, 2. Soils and vegetation with notes on Rodondo Island. Pap. Proc. R. Soc. Tasm. 107: 131-144.

Kirkiatrick, J.B., Gilledder, L., Hroke, J. and Harris, S., 1991: RESERVATION AND CONSERVATION STATUS OF TASMANIAN NATIVE HIGHER PLANTS. Wildl. Div. Sci. Rep. 91/2, Dep. Parks, Wildlife \& Heritage Tasm.

MACARTHLR, R.H. \& WIISON, E.O., 1967: THE THEORY OF ISLAND BIOGEOGRAPHY. Princeton University Press, Princeton.

MoMahon, A.R.G., Carr, G.W., Tomb, J.A. \& Rach, G.C., 1990: THE CONSERVATION STATUS OF MAIOR PLANT COMMUNITIES IN AUSTRALIA: VICTORIA. Ecological Horticulture Pty Ltd, Clifton Hill.

Marginson, M.A. \& Murray-Smith, S., 1969: Further investigations in the Kent Group (Bass Strait Islands). Vict. Nat. 86: 254-268.

Munfit, J.H. \& Murray-SMTth, S., 1967: First footing on a Bass Strait island: an investigation of Dover Is. in the Kent Group. Vict. Nat. 84: 239-250.

SCARIEIT, N.H., HOH, G.S. \& CADDER, D.M., 1974: The floristics and plant communities of the Hogan Group. Pap. Proc. R. Tasm. 107: 83-98.

SPHCHY, R.L., 1981: Conservation of vegetation types. In Groves, R.H. (Ed.): AUSTRAILAN VEGETATION. Cambridge University Press, Melbourne: 393-410. 